\title{
Adherence to Treatment Guidelines in Breast Cancer Care - a Retrospective Analysis of the 'Organgruppe Mamma der Arbeitsgemeinschaft Gynaekologische Onkologie'
}

\author{
Christian Jackisch $^{\mathrm{a}} \quad$ Michael Untch $^{\mathrm{b}}$ Dimitrios Chatsiproios ${ }^{\mathrm{c}}$ Christiane Lamparter $^{\mathrm{e}}$ \\ Friedrich Overkamp $^{d} \quad$ Werner Lichtenegger $^{f} \quad$ Wolfgang Rönsberg $^{g} \quad$ Christoph Thomssen $^{\mathrm{h}}$ \\ Gunter von Minckwitzi Ute-Susann Albert', on behalf of the 'Organgruppe Mamma \\ der Arbeitsgemeinschaft Gynäkologische Onkologie'*
}

${ }^{a}$ Clinic for Gynaecology and Obstetrics, Klinikum Offenbach GmbH; ${ }^{b} C l i n i c$ for Gynaecology and Obstetrics, Helios Klinikum Berlin Buch; ${ }^{c}$ CGG Klinik GmbH, Mannheim; dSurgery and Day-Care Unit for Oncology, Recklinghausen; ' MMF GmbH, Medical Research, Herdecke; f Department of Gynaecology Charité Berlin; 9Dr. Rönsberg GmbH, Munich; hDepartment of Obstetrics and Gynaecology, Martin-Luther University Halle; iGerman Breast Group, Neu-Isenburg; 'Department of Gynaecology, Gynaecologic Endocrinology and Oncology, University of Marburg, Germany

\section{Key Words}

Breast cancer treatment · Healthcare research · Adjuvant treatment . Breast cancer, metastatic - Guideline adherence

\section{Summary}

Background: The Organgruppe Mamma of the Arbeitsgemeinschaft Gynäkologische Onkologie (AGO) performed a nationwide 3-phase analysis of the structure of care and standard of therapy given to patients with breast cancer from 2002 (4th quarter) to 2004 (4th quarter). The extent to which national and international treatment recommendations are implemented in routine clinical practice had so far not been evaluated in an interdisciplinary approach. No reliable data on the pattern of care of these patients have been published in Germany before. Patients and Methods: The project included early breast cancer in the adjuvant and neoadjuvant setting as well as metastatic disease. We present the results of phase III of the AGO analysis, which are based on a survey conducted by the Organkommission Mamma in the 4th quarter of 2004. Results: Evaluation of the data reveals that treatment based on the guidelines is now being implemented very reliably in certain sectors. This is of particular relevance to the pattern of adjuvant treatment in early breast cancer. In contrast, in metastatic breast cancer (MBC), the complexity of the interdisciplinary treatment approach is complicating this kind of straightforward analysis. Conclusion: The present analysis conducted by the AGO was the first attempt to analyse the treatment provided in patients presenting with $\mathrm{MBC}$ in a systematic fashion. The fundamental problem remains, irrespective of the stage of the tumour, that too few patients are treated in randomised clinical trials. The mission set by the AGO-Organkommission Mamma is the longitudinal observation of the therapy practices for breast cancer on the basis of the observations discussed here, which should ultimately benefit the optimisation of therapy quality in Germany.

*Members of the Mamma Panel of the Gynaecological Oncology Association $(\mathrm{AGO})$ can be found at the end of the article.
Schlüsselwörter

Brustkrebsbehandlung · Untersuchung der Gesundheitspflege . Adjuvante Therapie - Brustkrebs, metastatischer - Einhaltung von Richtlinien

\section{Zusammenfassung}

Hintergrund: Die Organgruppe Mamma der Arbeitsgemeinschaft Gynäkologische Onkologie (AGO) hat eine landesweite 3-Phasen-Analyse zu Struktur und Standard der medizinischen Versorgung von Brustkrebspatienten im Zeitraum von 2002 (4. Quartal) bis 2004 (4. Quartal) durchgeführt. Das Ausmaß der Umsetzung nationaler und internationaler Behandlungsempfehlungen in der allgemeinen klinischen Praxis war bisher noch nie in einem interdisziplinären Ansatz bewertet worden, und bisher waren keine verlässlichen Daten zur Versorgungsstruktur dieser Patienten in Deutschland veröffentlicht worden. Patienten und Methoden: Das Projekt umfasste primären Brustkrebs in der adjuvanten und neoadjuvanten Situation sowie metastatischen Brustkrebs. Wir präsentieren die Ergebnisse der Phase III der AGO-Analyse, welche auf einem von der Organkommission Mamma im 4. Quartal des Jahres 2004 erstellten Gutachten basieren. Ergebnisse: Die Auswertung der Daten zeigt, dass in bestimmten Bereichen auf Richtlinien basierende Behandlungsansätze sehr verlässlich angewandt werden. Dies ist besonders relevant in Hinsicht auf die Struktur der adjuvanten Behandlung bei frühem Brustkrebs. Im Gegensatz dazu erschwert die Komplexität des interdisziplinären Behandlungsansatzes bei metastatischem Brustkrebs solch eine einfache Analyse. Schlussfolgerung: Die vorliegende AGO-Analyse war der erste Versuch, die Behandlung von Patientinnen mit metastatischem Brustkrebs systematisch zu analysieren. Ein grundlegendes Problem ist weiterhin, dass unabhängig vom Tumorstadium zu wenige Patienten im Rahmen randomisierter klinischer Studie behandelt werden. Die Mission der AGO-Organkommission Mamma ist die Langzeitüberwachung von gebräuchlichen Therapieansätzen bei Brustkrebs auf der Basis der hier diskutierten Beobachtungen, was letztendlich der Optimierung der Behandlungsqualität in Deutschland dienen sollte.

\begin{tabular}{ll}
\hline KARGER & ๑ 2008 S. Karger GmbH, Freiburg \\
Fax +49761 45207 14 & Accessible online at: \\
$\begin{array}{l}\text { E-mail Information@Karger.de } \\
\text { www.karger.com }\end{array}$ & www.karger.com/brc
\end{tabular}

Prof. Dr. med. Christian Jackisch

Klinikum Offenbach GmbH

Frauenklinik - Brustzentrum Offenbach

Starkenburgring 66, 63069 Offenbach, Germany

Tel. +49 69 8405-3805, Fax -4456

E-mail christian.jackisch@klinikum-offenbach.de 


\section{Introduction}

Awareness of the status quo in the pattern of treatment of breast cancer patients in Germany is the prerequisite for planning demand-oriented treatment opportunities, as well as for the implementation of care which would provide blanket coverage in compliance with the guidelines. Implementation of the course of treatment in accordance with the guidelines makes high demands on the interdisciplinary therapeutic community. This was the starting point for a quality assurance analysis by the Arbeitsgemeinschaft Gynäkologische Onkologie (AGO), which was conducted in 3 stages (phases). Phase I and II were dedicated to data validation which was followed by a prospective survey (phase III).

As part of phase I of the project (4th quarter 2002), an incidence of 50,344 patients with breast cancer in Germany was evaluated. This figure correlates with the data from the various specialist organisations and with the release of information by the Federal Statistical Office in Wiesbaden, Germany, and hence underlines the validity of the sampling procedure adopted for the AGO survey. The majority of patients were treated in the gynaecological wards of hospitals, more specifically in university hospitals. Whereas during the survey period, gynaecologists were almost exclusively responsible for primary treatment (surgical and systemic), the majority of metastatic patients found themselves in the care of internal medical/oncological specialists, particularly in specialist practices.

\section{Material and Methods}

The results of phase III of the AGO analysis, which are to be presented here, are based on a survey conducted in the 4th quarter of 2004. The survey was conducted by the Organkommission Mamma of the AGO in cooperation with the Working Group for Internal Medicine/Oncology (AIO), the Professional Association of Practising Gynaecological Oncologists (BNGO), and the North-Eastern German Society of Gynaecological Oncology (NOGGO). Mainly phase III of the AGO analysis is accordingly arranged as treatment documentation. The focus is on medicinal treatment as part of an interdisciplinary approach to treatment.

As part of a preliminary survey (so-called centre survey), letters were sent to 1,880 gynaecological and oncological clinical wards nationwide, as well as to gynaecological and clinical practices specialising in oncology that had diagnosed and were treating patients with breast cancer (gross sample). A total of $27.5 \%$ of respondents, i.e. 518 wards/practices, were willing to participate in the survey (net sample). The participating wards/ practices involved in care stage A + B (A: university hospitals, high-dependency care with central monitoring; B: specialist care) were required to have at least 30 patients, and those involved in care stage $\mathrm{C}$ (basic/ standard care) to have at least 15 patients with breast cancer who were undergoing treatment. Following this filter question, the data of 1,069 patients from 257 wards/practices were included in the main survey. All patients were required to have received at least 3 cycles of chemotherapy and/or antibody therapy. Distribution among the care stages and specialists groups correlated with the distribution calculated in phase II of the nationwide distribution, according to which the majority of breast cancer patients in Germany are treated by gynaecologists. Of the 1,069 patients in the random sample, 748 were being treated by gynaecologists, 115 in private practice.

\section{Results}

Adjuvant and Neoadjuvant Treatment of Early Breast Cancer $(E B C)$

\section{Patient Characteristics}

At the time of the main survey, 34\% (366 of 1,069) of patients documented were either in the adjuvant or neoadjuvant setting or entered the survey with metastatic breast cancer (MBC) $(66 \%)$. Mean age was 52 years. The average interval from initial diagnosis to project entry was 5.5 months (169 days). The majority of patients were postmenopausal (53\%). According to the treating physicians, $7 \%$ of the women had treatment-related concomitant diseases, $1 \%$ of which were cardiac disorders. The highest percentage of patients receiving neoadjuvant treatment was found to be in university hospitals and in high-dependency units with central monitoring, and was slightly higher among oncologists than gynaecologists (37 vs. $28 \%$ ).

\section{Primary Diagnosis}

Diagnosis was mainly (58\%) established by gynaeco-oncological clinicians or clinicians working in the field of oncology. At the time of the initial diagnosis, $13 \%$ of patients had stage IA, $51 \%$ stage IIA/B, and $12 \%$ stage IIIA. Advanced stage IIIB/C/IV were less than $20 \%$. The majority of tumours were poorly differentiated: 47 and $46 \%$ were G2 or G3 tumours, respectively. Nodal involvement was positive in $60 \%$. Endocrine responsive tumours were present in $70 \%$ of pre- and postmenopausal women with positive hormone receptor status (oestrogen receptor $(\mathrm{ER})+$ and/or progesterone receptor $(\mathrm{PR})+)$.

\section{Current Primary Treatment}

Of the patients treated with adjuvant therapy, $80 \%$ received chemotherapy, $70 \%$ endocrine treatment, and $79 \%$ radiotherapy. At the time of the survey, trastuzumab was rarely used in adjuvant or neoadjuvant therapies. The majority of women were treated as out-patients $(63 \%)$ or in a day-care unit (22\%). Only $4 \%$ of patients were hospitalised. Actual therapy decisions were based on the consensus recommendations of St. Gallen 2003, and on the therapeutic guidelines issued by specialist organisations, irrespective of the stage of care.

\section{Surgery}

Of the patients undergoing breast surgery, $90 \%$ received surgical interventions, including breast conserving therapy (BCT). Of this cohort, $90 \%$ underwent complete axillary dissection. A sentinel lymph node dissection (SLN) was documented in $15 \%$. Review of the pathology reports revealed clear margins in $95 \%$ ( $\mathrm{R} 0$ resection).

\section{Radiotherapy}

As part of their treatment, $73 \%$ of patients treated with adjuvant or neoadjuvant therapy received radiotherapy. Radio- 
therapy was predominately performed postoperatively (67\%); more specifically, in $83 \%$ after completion of chemotherapy. The chest wall ( 66 vs. $46 \%$ ) and lymphatic route (34 vs. $21 \%$ ) were more frequently included in the radiotherapy in patients undergoing mastectomy compared to BCT.

\section{Adjuvant and Neoadjuvant Chemotherapy}

Adjuvant chemotherapy was mostly administered using anthracycline- or alternatively anthracycline/taxane-based regimens. $68 \%$ of patients received an anthracycline-containing and $18 \%$ an anthracycline/taxane-containing chemotherapy. The CMF regimen (cyclophosphamide, methotrexate, 5-fluorouracil (5-FU)) was still being used in the adjuvant treatment of $9 \%$ of patients. This demonstrated a high level of adherence to the therapy recommendations of the AGO and the consensus decision of St. Gallen 2003 [1,2].

In the neoadjuvant treatment situation, only the anthracycline-containing or alternatively the taxane-containing regimen was applied, in accordance with the data status and the foregoing therapy recommendations, whereby the anthracycline/taxane-containing regimen at $61 \%$ - also in accordance with the data status - was the highest percentage. $38 \%$ of patients exclusively received an anthracycline-containing combination as neoadjuvant treatment. This also demonstrates a high degree of concordance with the therapy recommendations of the AGO and the St. Gallen conference. The use, in accordance with the guidelines, of the anthracycline or alternatively taxane-containing regimen in adjuvant/neoadjuvant therapy had also proved to be a distinct trend in the historical course of therapy in metastatic patients treated with preoperative adjuvant therapy.

An extensive evaluation of the database on the use of adjuvant chemotherapy reveals that anthracycline and taxane were used in all age groups. Elderly patients were also treated with anthracycline and/or taxane. The CMF regimen ceased to have any significance, in particular for patients with lymph node invasion; $3 \%$ of these patients were still receiving CMF. The majority of nodal negative patients $(80 \%)$ also received treatment with anthracyclines that were supplemented with taxane in some cases. The proportion of nodal positive patients treated with anthracyclines and/or taxanes was now $90 \%, 26 \%$ of whom were receiving combined anthracycline/taxane treatment.

\section{Participation in Clinical Trials}

Almost $30 \%$ of patients treated with neoadjuvant therapy and $15-20 \%$ of patients treated with adjuvant therapy were treated in the setting of a randomised clinical trial (RCT). Gynaecologists included more patients into RCT compared to medical oncologists. The gynaecological wards at university hospitals or academic hospitals listed highest in RCT participation (35\% RCT in the neoadjuvant, and 24\% RCT in the adjuvant setting). However, in the gynaecological wards of communitybased hospitals, trial participation was $31 \%$ for the neoadjuvant and $19 \%$ for the adjuvant setting.

\section{Endocrine Treatment}

Over time, $82 \%$ of patients with endocrine responsive disease received adjuvant antihormonal therapy at the time of documentation; endocrine treatment was planned for a further $11 \%$. The majority of patients $(88 \%)$ received sequential chemo-endocrine therapy in accordance with the current treatment recommendations. As documented, the treatment of choice was tamoxifen in $82 \%$. Aromatase inhibitors - commensurate with the data status at that time - played a negligible role in adjuvant therapy. 53\% of premenopausal patients received gonadotropin-releasing hormone $(\mathrm{GnRH})$ analogues, mostly in combination with tamoxifen.

In conclusion the survey revealed that the majority of patients was treated with a high adherence to the available therapy guidelines. BCT was performed in more than $60 \%$. Implementation of the guideline recommendations in the application of an anthracycline-based adjuvant chemotherapy for administration in a risk-adapted combination with taxane deserves a special mention.

\section{Treatment of Metastatic Breast Cancer}

\section{Patient Characteristics}

Less than two thirds of patients $(66 \%)$ included in the main survey had MBC at the time of the survey (703 of 1,069). Average age was 58 (range $22-84$ years). Specifically, $15 \%$ of patients were $\geq 70$ years old, and three quarters of patients were at least 50 years old. Two thirds of metastatic patients were postmenopausal. Despite the advanced age in some cases, the Karnofsky index was $83.6 \%$, and no substantial differences associated with the line of treatment were evident. This shows good tolerance of the treatment. Only $6 \%$ of patients had a clinically relevant associated disease. Patients were in different lines of treatment. A percentage of patients had additionally received adjuvant pretreatment. On the documentation date, the majority of patients were receiving first- or second-line treatment (37 and $29 \%$, respectively). Over a third of the women (234 of 703) were receiving third- or fourth-line therapy. All age groups were represented in all 4 lines of treatment, which proves, among other things, the patients' willingness to receive therapy, which was also evident among older patients.

\section{Primary Diagnosis and Course of Treatment}

Almost all patients with MBC were initially diagnosed with 'breast cancer' in hospitals. In general, 20\% ( $\mathrm{n}=137)$ were at stage IV at the time of diagnosis. $60 \%$ of patients were diagnosed at stage I/II. Poor grading was more apparent than was normally the case: $51 \%$ of patients who have since become metastatic had a G3 tumour upon initial diagnosis, and a fur- 
ther $38 \%$ a G2 tumour. $68 \%$ of patients had lymph node invasion, and $64 \%$ hormone-sensitive breast cancer (positive hormone receptor status: $\mathrm{ER}+$ and/or $\mathrm{PR}+$ ). HER2 receptor status testing had already been performed in $64 \%$ of patients. In this context, substantial differences were demonstrated between patients diagnosed prior to and after 1999. After 1999, the proportion of HER2 receptor status tests increased substantially to $85 \%$. Further prognosis of the patients correlated with the prognostic factors in the initial diagnosis. Patients without lymph node invasion invariably had a better prognosis than patients with lymph node invasion.

\section{Treatment prior to $M B C$}

The vast majority of patients at the metastatic stage received adjuvant or neoadjuvant treatment. This also applied to the metastatic situation. A total of $73 \%$ of patients received chemo-therapy as part of adjuvant, or alternatively neoadjuvant, primary therapy, $60 \%$ radiotherapy, and $48 \%$ antihormonal treatment. The data indicate that antihormonal therapy was underrepresented as the initial therapeutic approach since two thirds of patients had a hormone-sensitive tumour.

\section{Surgery and Radiation as part of Primary Treatment prior to $M B C$}

Breast conserving surgery as part of primary therapy showed a substantial increase during the period of 1994-2003. Radiotherapy was generally performed postoperatively as part of primary therapy $(80 \%)$ and in $50 \%$ of patients after chemotherapy. The sandwich procedure (51 vs. $6 \%$ ), radiation of the thoracic wall (71 vs. $46 \%$ ) and of the efferent lymph paths (55\% vs. $31 \%$ ) were performed more frequently in mastectomised patients than in BCT patients.

\section{Primary Chemotherapy}

The younger the patients, the more frequently adjuvant or neoadjuvant chemotherapy was applied. For patients $\geq 80$ years of age, the focus was on antihormonal therapy at $60 \%$, but $20 \%$ of these women were also treated with chemotherapy. The negligible number of study participants was conspicuous. At that time, only $6 \%$ of patients were receiving adjuvant therapy as part of a clinical study, against $27 \%$ of patients who were receiving neoadjuvant therapy.

Retrospective consideration of substances used as part of primary therapy has shown a substantial increase in an anthracycline-containing and anthracycline/taxane-containing regimens since the end of the 1990s. This was accompanied by a considerable decline in the use of the $\mathrm{CMF}$ regimen. This demonstrates that treating physicians increasingly base their therapy decisions on the latest study data and on therapy recommendations. The trend in favour of the use of an anthracycline-containing or alternatively anthracycline/taxane-containing regimen also appears to be linked to the increased use of neoadjuvant chemotherapies. In neoadjuvant therapy, an anthracycline-containing or alternatively anthracycline/taxane-

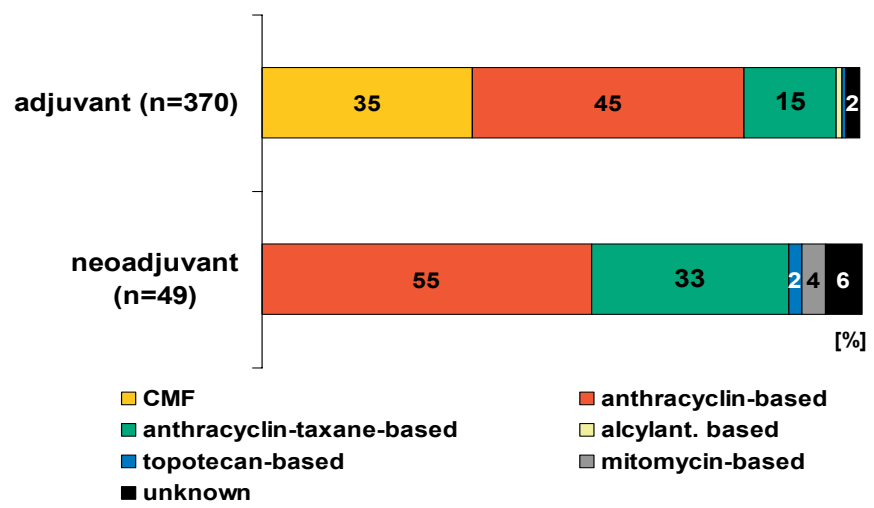

Fig. 1. Timing of chemotherapy (neoadjuvant vs. adjuvant) and the chemotherapeutic agents used in either setting.

containing regimen was used almost exclusively since the success of this therapeutic approach is based on study data with these substances. Dose reductions and prolonged intervals between them were documented in fewer than $10 \%$ of therapies. The timing of chemotherapeutic intervention (primary systemic vs. adjuvant) determines the cytostatics used (fig. 1).

\section{Endocrine Treatment as Part of Adjuvant Therapy}

Seventyfour \% of metastatic patients who had previously received adjuvant therapy were found to have positive hormone receptor status (ER+ and/or $\mathrm{PR}+)$. However, only $48 \%$ of these patients were receiving antihormonal therapy. If antihormonal therapy was performed, it took place - in accordance with the guidelines - in $81 \%$ of cases after adjuvant chemotherapy. Almost all patients $(88 \%)$ received treatment with tamoxifen which was the standard at that time.

\section{Adjuvant use of Trastuzumab}

An adjuvant antibody therapy with trastuzumab was only possible at that time as part of a clinical study. Accordingly, only $2 \%$ of patients received adjuvant trastuzumab treatment; based on the number of patients with positive HER2 receptor status, this accounted for $7 \%$. The situation was similar for adjuvant bisphosphonate therapy.

\section{Current Treatment of the Metastatic Disease}

Almost all patients with $\mathrm{MBC}$ received chemotherapy while being entered in this project. Less than $50 \%$ received endocrine therapy. Only a minority received trastuzumab. Objective remissions were higher in patients receiving a combination chemotherapy than in those treated with monotherapy (41 vs. 34\%). This trend was irrespective of the line of treatment. Combination chemotherapy was preferred in first-line treatment of MBC. In this context, half of the patients received a combination therapy or monotherapy, respectively, irrespective of the number of metastatic sites. In the other lines of treatment, the percentage of combination therapies fell to around $20 \%$. Irrespective of the number of organs involved in $\mathrm{MBC}$, a trend in favour of chemotherapy was evident in first- 


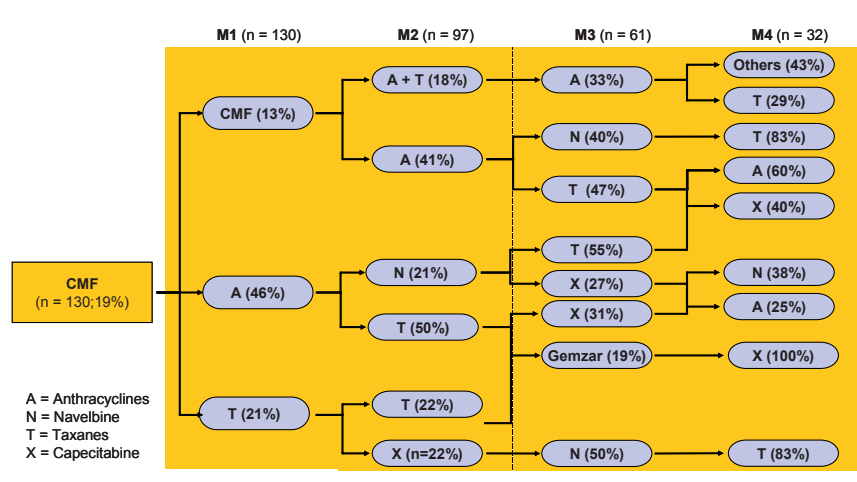

Fig. 2. Metastatic breast cancer therapy cluster for patients pre-treated with $\mathrm{CMF}$ in the adjuvant setting. Evaluation based on 703 patients in this survey.

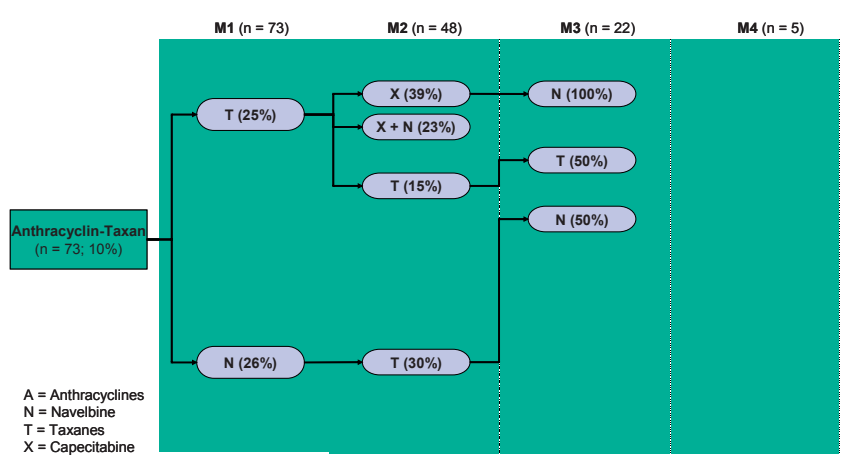

Fig. 4. Metastatic breast cancer therapy cluster for patients pre-treated with anthracyclines and taxanes in the adjuvant setting. Evaluation based on 703 patients in this survey.

line treatment. Hardly any patient received antihormonal treatment only or alternatively antibody therapy. Differences in the therapy decision in the first-line situation were not apparent for patients with visceral or alternatively non-visceral metastases. Postmenopausal patients more frequently received chemotherapy exclusively as a first-line treatment than premenopausal women. In terms of the various stages of care, the therapy decisions made for premenopausal patients were far more heterogeneous.

\section{Endocrine Treatment}

Only $48 \%$ of patients with MBC presenting with positive hormone receptor status $(\mathrm{ER}+$ and/or $\mathrm{PR}+)$ received antihormonal therapy, irrespective of the line of treatment.

\section{HER2 Status Testing and Therapy with Trastuzumab}

HER2 status was known in $80 \%$ of patients presenting with MBC. Hospital gynaecologists evaluate the HER2 status more frequently (81-88\%) than hospital oncologists (59- 75\%). HER2 status had been evaluated for $85 \%$ of patients even in the gynaecological wards in units providing basic, standard care. In the private sector, the rate of HER2 determination was encouragingly high at $87 \%$ for gynaecologists and $86 \%$ for oncologists.

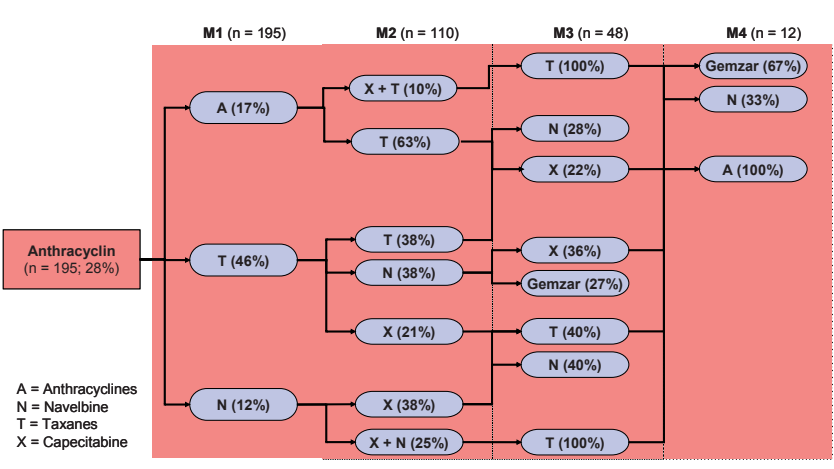

Fig. 3. Metastatic breast cancer therapy cluster for patients pre-treated with anthracyclines in the adjuvant setting. Evaluation based on 703 patients in this survey.

\section{Therapy Cluster for the Application of Chemotherapy in $M B C$} Treatment of MBC patients was performed within an RCT in $6 \%$ only. At university departments or academic institutions, the rate increased to $16 \%$. In contrast, clinics that provided basic, standard care did not include any patients in clinical studies. The most frequently used chemotherapeutic drugs in the metastatic setting were taxanes (23\% docetaxel, $17 \%$ paclitaxel), vinorelbine (22\%) in addition to capecitabine (15\%), epirubicin (14\%), and cyclophosphamide (13\%). The cytotoxic therapy sequence was dependent on the pretreatment of patients as part of their adjuvant therapy. Therapy clusters (M1: first line in $\mathrm{MBC}, \mathrm{M} 2$ : second line in $\mathrm{MBC}, \mathrm{M} 3$ : third line in MBC, M4: fourth line in MBC) were created for the metastatic setting for the first time on the basis of the data collected and in correlation with the primary therapy for the purpose of structuring the complexity in the treatment of MBC.

It was demonstrated that the most effective drugs were used either as monotherapy or combination therapy during treatment, irrespective of the type of pretreatment given. Chemotherapy-naive patients and those who had been pretreated with CMF, mainly received anthracycline/cyclophosphamide, or alternatively an anthracycline/taxane-containing regimen, as part of the first-line treatment (M1). Vinorelbine and capecitabine were important substances for subsequent lines of treatment. A reinduction of anthracyclines and taxanes, mostly as monotherapy, was a completely feasible option for a sufficiently long relapse-free period (fig. 2). Patients pretreated with anthracycline, subsequently received taxanes or alternatively taxane-containing therapy as a primary treatment (fig. 3). Alternatively, there was a reinduction of anthracyclines. Capecitabine, vinorelbine, and gemcitabine were potential options for the further therapy sequence as combination therapy or monotherapy, depending on the individual setting of the disease treated. The same treatment patterns were observed in patients previously treated with anthracyclines and taxanes as part of the primary therapy (fig. 4). Vinorelbine and cape-citabine were next in line as a therapy option, alternatively, reinduction with taxane was also an option in this context, in particular in the event of a sufficiently long relapse- 
free interval, for example, if other stages of therapy had been started in the meantime and there was another relapse. Substances that were used as part of last-line treatments were, among others, gemcitabine, 5-FU, alkylating agents, liposomal doxorubicin, bendamustine, topotecan, and platinum derivatives.

\section{Discussion}

The analysis presented here only highlights the treatment situation in Germany. Breast cancer therapy in RCT is, irrespective of the stage of the disease, predominantly performed at university hospitals and academic institutions. It is noteworthy that the same observations on the pattern of care in ovarian cancer have been reviewed by duBois et al. [3].

Timing of systemic therapy (primary systemic vs. adjuvant) had a considerable effect on the choice of the drugs used in operable breast cancer [4]. The observation that $30 \%$ of therapy in RCT was actually primary systemic therapy is encouraging, whereas it only comprised $15-20 \%$ in the adjuvant situation. With regard to the implementation of the national and international therapy recommendations and guidelines, an impressive, realistic implementation, particularly for the majority of patients treated outside clinical studies, irrespective of the place of care, has been ascertained. We learned that the issue of endocrine treatment in $\mathrm{MBC}$ needs ongoing medical education to ensure proper application of evidence-based treatment recommendations [5].

High-quality therapy results from the fact that dose reductions or interval prolongation were stated in less than $10 \%$ of cases, which implies a high relative dose intensity of the chemotherapy conducted. This is an important indicator for the quality of therapy [6]. The high rate of available predictive factors (ER, PR HER2) is proof of a positive implementation of an evidence-based approach. This is an important improvement in the treatment decision-making process in low risk populations [7]. A recent retrospective French report supports the use of multiple lines of chemotherapy in $\mathrm{MBC}$, suggesting that the time to progression or the duration of response is a clinically useful pattern for deciding to continue to treat $\mathrm{MBC}$ with multiple lines of chemotherapy [8]. This also applies to the therapy clusters from different lines of therapy for $\mathrm{MBC}$, demonstrating a high level of compliance to the AGO recommendations available at this time.

There is doubtlessly room to improve endocrine therapy, in particular for older patients who demonstrate an unexpectedly high willingness to undergo therapy during all stages of treatment of $\mathrm{MBC}$, even beyond the age of 70. It is a matter of reviewing the indication thoroughly and of also maximising supportive therapy. This applies in particular to adjuvant therapy in this age group [9]. The mission set by the AGO-Organkommission Mamma is to contribute to improvement of therapy quality in Germany by longitudinal observation of these therapy practices for breast cancer on the basis of the observations discussed here, which should also ultimately benefit the optimisation of therapy quality in Germany. Re-evaluation is scheduled for $2007 / 2008$.

\section{Acknowledgement}

The quality assurance programme of the AGO-Organkommission Mamma was realised by an unrestricted educational grant by BristolMyers-Squibb Germany, Essex Pharma Germany, und Roche Pharma Germany. The subject matter of the project was implemented independently and has no commercial objectives.

*Members of the Mamma Panel of the Gynaecological Oncology Association (AGO): Ute-Susann Albert, Marburg, Ingo Bauerfeind, München; Joachim Bischoff, Magdeburg; Jens Uwe Blohmer, Berlin; Klaus Brunnert, Osnabrück; Serban D. Costa, Magdeburg; Peter Dall, Lüneburg; Ingo J. Diel, Mannheim; Nikos Fersis, Chemnitz; Michael Friedrich, Krefeld; Kay Friedrichs, Hamburg; Bernd Gerber, Rostock; Uwe-Jochen Göhring, Bonn; Volker Hanf, Fürth; Nadia Harbeck, München; Jens Huober, St. Gallen; Christian Jackisch, Offenbach; Wolfgang Janni, München; Walter Jonat, Kiel (DKH); Manfred Kaufmann, Frankfurt; Hans-Joachim Hannover, Wiesbaden; Nicolai Maass, Kiel; Volker Möbus, Frankfurt; Ulrike Nitz, Mönchengladbach; Carsten Oberhoff, Essen; Mahdi Rezai, Düsseldorf; Gerhard Schaller, München; Anton Scharl, Amberg; Rita Schmutzler, Köln; Andreas Schneeweiss, Heidelberg (AIO); Ingrid Schreer, Kiel (DGS); Hans H. Kreipe, Hannover (DGP); Peter Sinn, Heidelberg (Pathologie); Erich F. Solomayer, Tübingen; Rainer Souchon, Hagen (ARO); Elmar Stickeler, Freiburg; Christoph Thomssen, Halle (Sprecher der AGO-Kommission Mamma); Michael Untch, Berlin; Gunter von Minckwitz, Neu-Isenburg/Frankfurt.

\section{References}

1 Goldhirsch A, Wood WC, Gelber RD, et al.: Meeting highlights: updated international expert consensus on the primary therapy of early breast cancer. J Clin Oncol 2003;21:3357-3365.

2 Von Minckwitz G, Brunnert K, Costa SD, et al.: Evidence based recommendations on primary treatment recommendations of carcinomas of the breast. Zentralbl Gynakol 2002;124:293-303.

3 DuBois A, Rochon J, Lamparter C, et al.: Pattern of care and impact of participation in clinical studies on the outcome in ovarian cancer. Int J Gynecol Cancer 2005;15:183-191.
4 Kaufmann M, Hortobagyi GN, Goldhirsch A, et al.: Recommendations from an international expert panel on the use of neoadjuvant (primary) systemic treatment of operable breast cancer: an update. J Clin Oncol 2006;24:1940-1949.

5 Von Minckwitz G: Evidence-based treatment of metastatic breast cancer - 2006 recommendations by the AGO Breast Commission. Eur J Cancer 2006:42:2897-2908.

-6 Schwenkglenks M, Jackisch C, Constenla M, et al.: Neutropenic event risk and impaired chemotherapy delivery in six European audits of breast cancer treatment. Support Care Cancer 2006;14:901-909.
7 Hebert-Croteau N, Brisson J, Latreille J, et al.: Compliance with consensus recommendations for systemic therapy associated with improved survival of women with node-negative breast cancer. J Clin Oncol 2003;21:3357-3365.

8 Dufresne A, Pivot X, Turnigand C, et al.: Impact of chemotherapy beyond the first line in patients with metastatic breast cancer. Breast Cancer Res Treat 2008;8:219-225.

9 Muss H, Woolf S, Berry D, et al.: Adjuvant chemotherapy in older and younger women with lymph node-positive breast cancer. JAMA 2005;293: 1073-1081. 